\title{
Benefícios da Estimulaçao Elétrica Nervosa Transcutânea para a redução da dor no pós-operatório da cesariana e sua relação com as limitações de atividade
}

\author{
Fábia Maria de Santana ${ }^{1}$; Emanuella Grangeiro Cavalcante ${ }^{2}$ Danielly M. G. Matias ${ }^{3}$; \\ Alessandro Adamo Gonçalves Oliveira ${ }^{4}$
}

\begin{abstract}
Resumo: A lesão tecidual causada por procedimentos cirúrgicos, e a consequente reação inflamatória decorrente deste processo, resultam em condições dolorosas no período pós-operatório. A dor pós-operatória de uma cesariana costuma retardar o retorno das puérperas às suas atividades de vida diária proporcionando-lhes diminuição da mobilidade e consequentemente limitações. A Estimulação Elétrica Nervosa Transcutânea (EENT) vem se apresentando, nos últimos anos, como um importante instrumento clínico no tratamento da dor. Objetivo: Avaliar o efeito da aplicação da EENT convencional para o alívio da dor em puérperas pós-cesariana. Método: Trata-se de uma pesquisa do tipo quantitativa de caráter experimental. A amostra constou de 20 puérperas, submetidas à cirurgia de cesariana, estando no puerpério imediato, apresentando dor no local da incisão cirúrgica. Para realização da coleta, foi utilizado um questionário de Limitação Funcional, um aparelho de Eletroestimulação Nervosa Transcutânea (EENT), e a Escala Visual Analógica (EVA) como meio de mensurar a dor antes e após a eletroestimulação. Resultados: Houve uma diferença estatisticamente significante entre as intensidades da dor antes e após o uso da EENT, onde a média encontrada antes da aplicação foi 6,35 e depois da aplicação 2,9. As principais limitações encontradas em consequência da dor pós-operatória foram para as funções de sentar e levantar da cama (90\%), ficar na posição de decúbito lateral (90\%) e tossir (85\%). Conclusão: A EENT mostrou-se ser eficaz, pois reduziu significativamente os escores da dor causados pela lesão tecidual.
\end{abstract}

Palavras-chave: Dor, Cesariana, EENT

\section{Benefits of Transcutaneous Electrical Nerve Stimulation for pain reduction in postoperative period of cesarean and its relation with activity limitations}

\begin{abstract}
The tissue injury caused by surgical procedures, and the consequent inflammatory reaction resulting from this process, resulting in painful conditions in the postoperative period. The postoperative pain is usually a cesarean delay the return of mothers to their activities of daily living by providing them with decreased mobility and consequently limitations. The Transcutaneous Electrical Nerve Stimulation (TENS) has been presented in recent years, as an important clinical tool in the treatment of pain. Objective: To evaluate the effect of the TENS conventional pain relief in postpartum post-cesarean section. Methods: This is a survey of the quantitative type of experimental character. The sample consisted of 20 mothers who underwent cesarean surgery, being in the immediate postpartum period, with pain at the surgical incision. For blood sampling, a questionnaire was used for Functional Limitation, a device for Transcutaneous Electrical Stimulation (TENS), and Visual Analogue Scale (VAS) as a means of measuring pain before and after stimulation. Results: There was a statistically significant difference between the intensities of pain before and after the use of TENS, which found the average was 6.35 before application and after application 2.9. The main limitations encountered as a result of postoperative pain for the functions were to sit down and get out of bed $(90 \%)$, stay in the lateral decubitus position $(90 \%)$ and cough $(85 \%)$. Conclusion: The TENS proved to be effective, since scores significantly reduced pain caused by tissue injury.
\end{abstract}

Keywords: Pain, Cesarean, TENS

\footnotetext{
${ }^{1}$ Mestranda, Ciências da Saúde, Faculdade de Medicina do ABC, Santo André, SP, Brasil. E-mail: fabiagarban@hotmail.com.

${ }^{2}$ Fisioterapeuta, pós graduada em Respiratória e UTI, pela unidade de treinamento São Camilo.

${ }^{3}$ Fisioterapeuta, graduada pela Universidade Federal da Paraíba e formação em Reeducação Postural Global.






\section{Introdução}

O período após o parto é chamado de puerpério, e acontece quando as modificações locais e sistêmicas provocadas no organismo da mulher pela gravidez e parto retornam ao estado prégravídico. Pode ser dividido em: puerpério imediato (do $1^{\circ}$ ao $10^{\circ}$ dia), tardio (do $10^{\circ}$ ao $45^{\circ}$ dia) e remoto (após o $45^{\circ}$ dia). O puerpério imediato é um momento caracterizado por importantes transformações físicas e emocionais. Os diversos desconfortos presentes nessa fase são inerentes ao processo de parturição, tais como: flatulência, dor no local da incisão da cirurgia, constipação intestinal, cólicas abdominais, incontinência urinária, posturas antálgicas e fraqueza abdominal devido à diástase do músculo reto abdominal (RETT et al., 2008; SANTANA et al., 2011).

É inevitável que o procedimento cirúrgico cause lesão tecidual. A presença da dor após a intervenção causa desconforto ao paciente, impedindo seu relaxamento e prejudicando sua movimentação no leito. A dor pós-operatória de uma cesariana costuma retardar o retorno dos indivíduos às suas atividades de vida diária, dificultando a recuperação e o contato da mãe com o recém- nascido, além de ser obstáculo ao bom posicionamento para a amamentação, o autocuidado e para realizar atividades cotidianas, como sentar e levantar, deambular, realizar higiene íntima, entre outras (TONELLA; ARAÚJO; SILVA, 2006; SOUSA et al., 2009).

A utilização de correntes elétricas terapêuticas constitui um dos vários recursos utilizado pela fisioterapia para reduzir a dor após a cirurgia cesariana, podendo atuar em diferentes condições, tais como: promover analgesia, melhoria do fluxo circulatório local, drenagem de líquidos, tonificação ou relaxamento muscular. Dos recursos disponíveis para aumentar a tolerância de indivíduos a esses estímulos dolorosos, a Estimulação Elétrica Nervosa Trancutânea (EENT), vem se apresentando, nos últimos anos, como um importante instrumento clínico no tratamento da dor (MELO et al., 2006; MACIEL; CAMARA, 2008).

A EENT é um recurso terapêutico seguro, pois se trata de uma corrente despolarizada, ou seja, a corrente elétrica gerada não causa queimaduras. A eletroestimulação convencional gera impulsos de curta duração e baixa amplitude. A intensidade da corrente é agradável e não gera contração muscular. Este tipo de aplicação é comumente indicado para o controle da dor aguda, como é o caso da dor pósoperatória. (SOUSA et al., 2009; FERREIRA; BELEZA, 2007).

A corrente produz analgesia através da ativação de receptores sensoriais periféricos, atuando através do fenômeno das comportas da dor, e aumentando a produção de endorfinas. Segundo essa teoria, há um bloqueio dos impulsos da dor que vêm da periferia. Esse bloqueio na substância gelatinosa agiria como uma comporta que tem a capacidade de impedir que impulsos aferentes alcancem as células $\mathrm{T}$, as quais conduzem a sensação de dor para diferentes partes do cérebro, 
cerebelo, tronco cerebral, tálamo e córtex (BAVARESCO et al., 2011; FERRAZ; MOREIRA, 2009).

Este recurso se apresenta vantajoso por ser de baixo custo e não apresentar efeitos colaterais.

Diante dessas vantagens a EENT tem sido introduzida em hospitais pela fácil utilização e uma menor necessidade de administração de fármacos, promovendo assim o bem-estar do paciente e a redução de custos (TELLES et al., 2011).

O uso da EENT pode diminuir o consumo de analgésicos pelos pacientes, bem como reduzir os níveis de dor avaliados por EVA (Escala Visual Analógica), outro benefício é a possibilidade da movimentação precoce, facilitada pela redução da dor propiciada pelo seu uso. Diante disso, o presente estudo objetivou avaliar o efeito da aplicação da EENT convencional para o alívio do quadro álgico em puérperas pós-cesariana, por meio da EVA, e verificar quais as principais atividades funcionais que causam um maior desconforto e lhe proporcionam uma maior limitação.

\section{Método}

O delineamento adotado foi uma pesquisa do tipo experimental com abordagem quantitativa. As pacientes foram informadas sobre o objetivo e benefícios do estudo e aceitaram participar voluntariamente de acordo com o que determina a Resolução n. 196/96 do Conselho Nacional de Saúde, envolvendo seres humanos.

A pesquisa foi realizada na Enfermaria Obstétrica do Alojamento Conjunto do Hospital Regional de Sousa (HRS), onde a população do estudo foi composta por 20 puérperas, submetidas à intervenção cirúrgica de cesariana. Como critérios de inclusão foram considerados que a puérpera deveria estar no puerpério imediato e apresentar uma intensidade de dor superior à zero classificada através da EVA. Foram excluídas da pesquisa puérperas que estivessem com um tempo inferior a 12 horas da anestesia, ou que já tivessem realizado mais de duas cesarianas.

Foram utilizados para determinada pesquisa: um Questionário de Limitação Funcional, uma Escala Visual Analógica e um aparelho de Eletroterapia Portátil. O questionário foi composto por 10 perguntas, e teve como objetivo verificar as principais limitações encontradas nas puérperas em decorrência da dor pós-operatória, relacionadas à mobilidade (sentar, levantar, deambular), ao autocuidado (urinar) e ao cuidado com o bebê (amamentar).

A EVA é um instrumento de fácil compreensão, formada por uma reta horizontal de $10 \mathrm{~cm}$, sendo dividida da seguinte forma, dor leve ( 0 a 2), dor moderada ( 3 a 7 ) e dor intensa ( 8 a 10), e serviu como parâmetro para que a puérpera pudesse quantificar a dor referida antes e após a aplicação da EENT. O aparelho eletroestimulador utilizado foi o Neurodyn Portable TENS/FES da marca Ibramed, onde a corrente utilizada foi a convencional $(\mathrm{F}=100 \mathrm{~Hz}$ e $\mathrm{T}=501 \mathrm{~s})$. 
Durante sua realização, foram utilizados quatro eletrodos de borracha, gel condutor, e uma fita adesiva antialérgica para fixá-los. Os eletrodos foram posicionados na forma peri-incisional, técnica bipolar cruzada na região de baixo ventre. A intervenção durou 30 minutos.

A coleta de dados foi realizada no mês de Maio de 2012. A pesquisa foi feita durante o período de puerpério imediato, estando no $2^{\circ}$ DPO de internação obstétrica, duas vezes por semana, de forma individual e com uma linguagem de fácil compreensão.

O Processamento dos dados foi realizado pela estatística descritiva simples, correlacionando os dados coletados demonstrado em gráficos para melhor compreensão do leitor. Para a realização da análise dos dados foi utilizado o Microsoft Office Excel 2010 para correlacionar os dados do questionário e o método $t$ - student para verificar o nível de significância da dor.

\section{Resultados}

Para realização do presente estudo, foi selecionada uma amostra de 20 mulheres submetidas à cesariana no período de puerpério imediato. Para comparar a intensidade da dor e quantificá-la através da EVA, foi utilizado o método $t$-student, onde a média encontrada para intensidade antes da aplicação da EENT foi de 6,35 e a média encontrada para classificar a dor após seu uso foi de 2,9. O nível de significância para o teste $t$-student foi de $5 \%$.

Vale salientar que as intensidades da dor de acordo com a EVA, são classificadas em leve, moderada ou intensa.

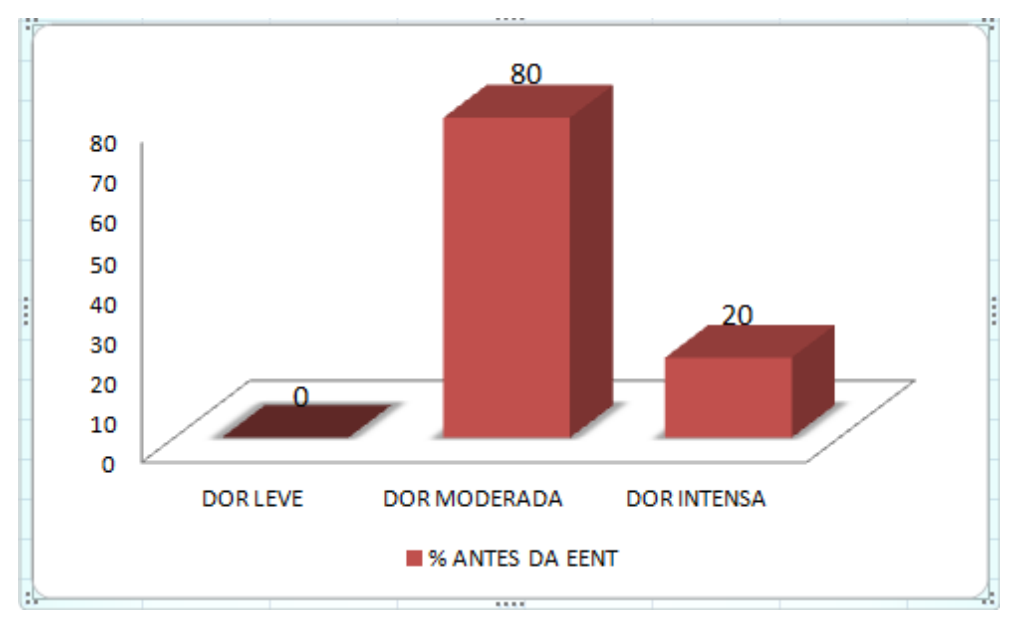

Gráfico 1- Caracterização da dor pela EVA antes da EENT 
Diante disso, de acordo com o gráfico 1, é importante observar que antes da eletroestimulação, nenhuma puérpera, $0 \%$ relatou sentir dor leve. Um total de $80 \%$, que caracterizou o maior percentual de intensidade foi atribuído para dor moderada. A dor intensa estava presente em $20 \%$ das puérperas.

Ao analisar os níveis da dor após a utilização da EENT, é possível observar que houve uma redução significativa dos seus níveis em comparação a antes do seu uso.

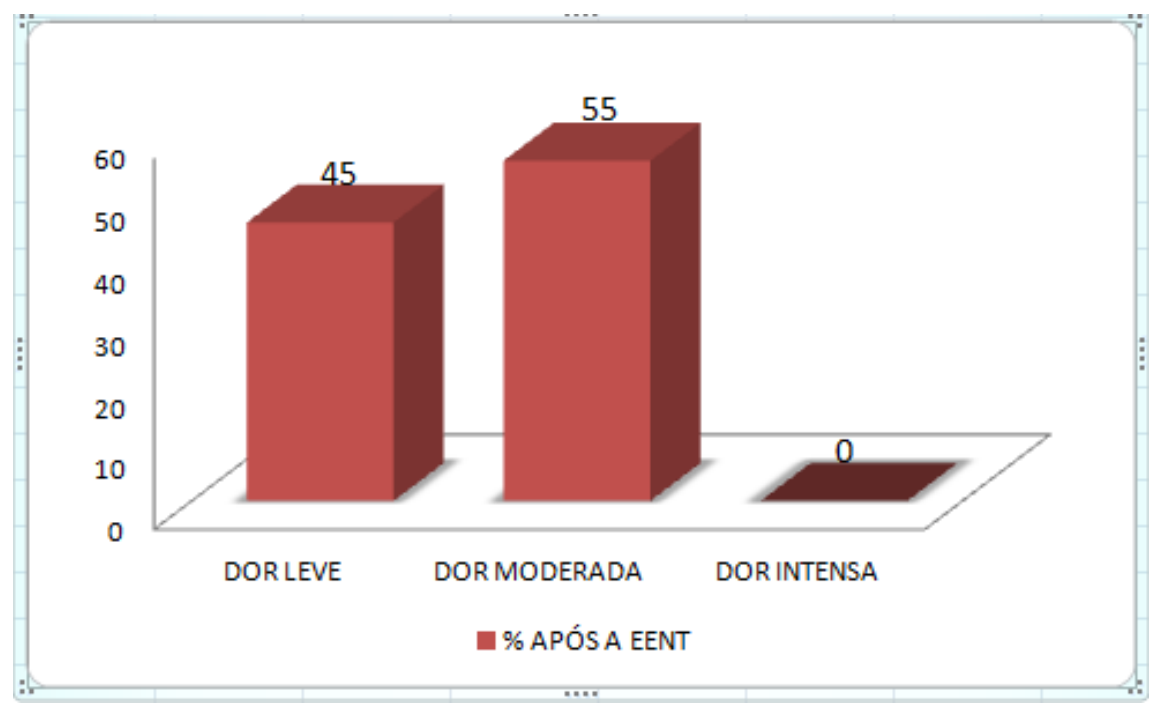

Gráfico 2- Caracterização da dor pela EVA após o uso da EENT

Como pode ser observada no gráfico 2, o escore de dor intensa foi abolido após a aplicação da EENT (0\%). O escore de dor moderada foi reduzido de $80 \%$ para $55 \%$ após a eletroestimulação. A dor leve correspondeu a $45 \%$ da amostra.

Para caracterizar as principais limitações proporcionadas pela dor pós-operatória, foi utilizado um questionário de limitação funcional, que teve como objetivo avaliar as principais limitações de atividades apresentadas pelas puérperas, como sentar e levantar, deambular, amamentar, tossir, dentre outras. O gráfico 3 apresenta a distribuição da limitações que apresentaram-se com maior frequência ocasionadas pela dor devido a incisão cirúrgica. 


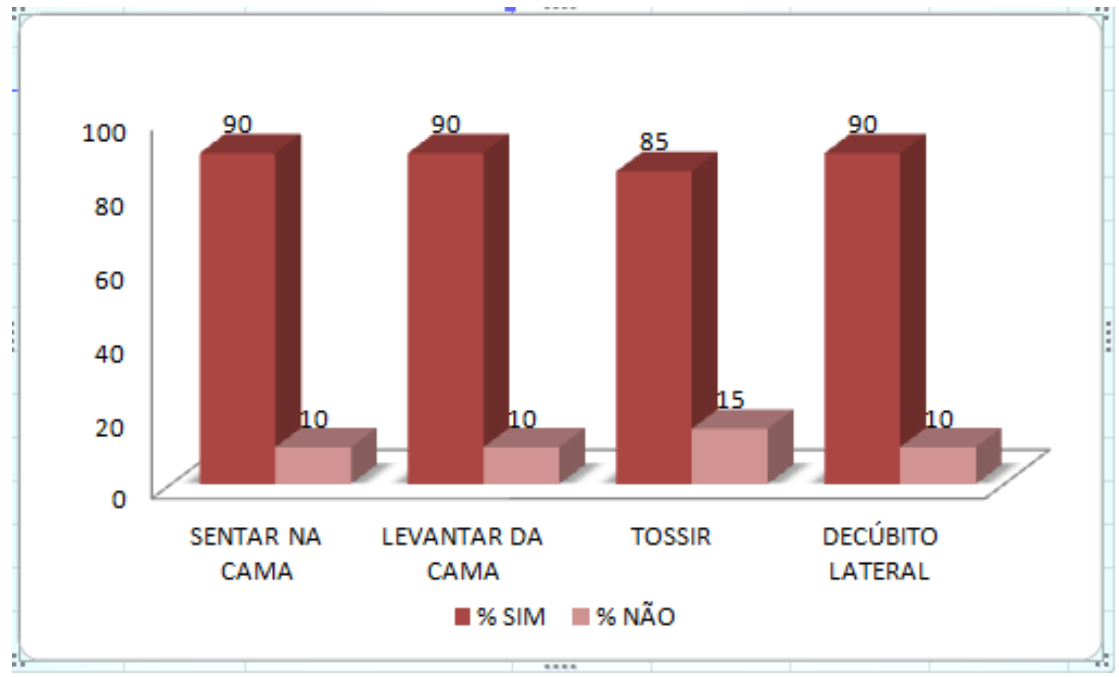

Gráfico 3- Distribuição das principais limitações funcionais

Quando questionadas sobre a presença de limitações nas funções de sentar e levantar da cama, $90 \%$ das puérperas afirmaram que apresentavam uma sensação de desconforto. Já no que diz respeito à tosse, um percentual de $85 \%$ referiu apresentar dor durante o seu ato. Durante a mudança de decúbito dorsal para decúbito lateral, $90 \%$ das puérperas afirmaram apresentar dor.

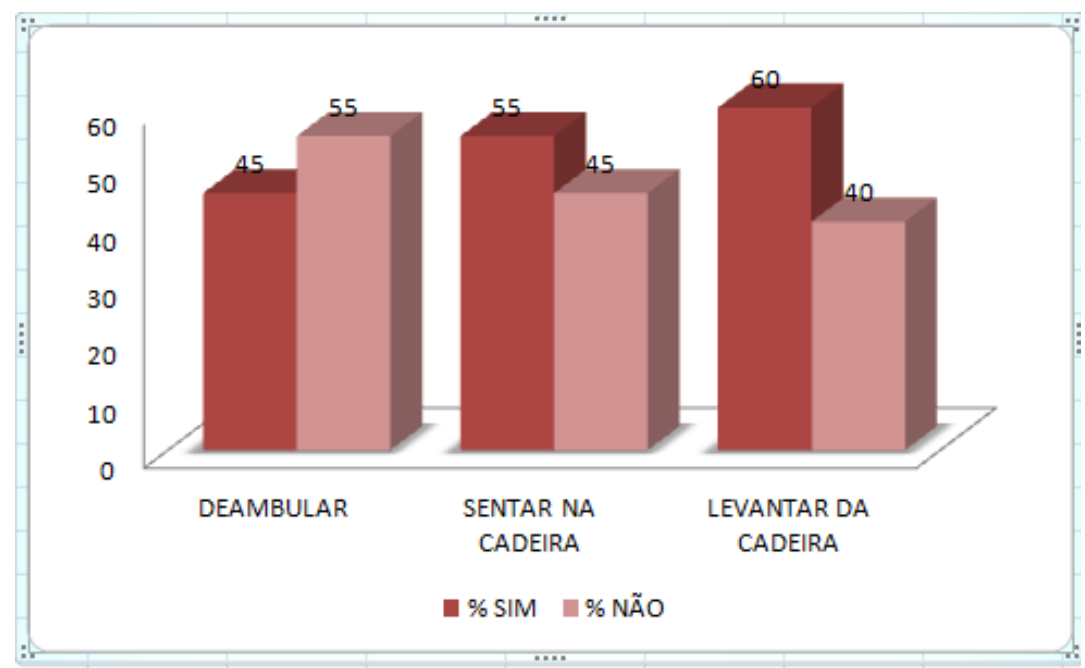

Gráfico 4- Distribuição das principais limitações

Como pode ser observado no gráfico 4 , um menor escore de dor foi encontrado durante a atividade de deambular, caracterizando um percentual de $45 \%$. Ao avaliar a função de sentar na cadeira, 55\% apresentou desconforto durante essa atividade. Com relação à atividade de levantar da cadeira, $60 \%$ das puérperas referiu sentir dor. 




Gráfico 5- Distribuição das principais limitações

As menores percepções dolorosas podem ser analisados através do gráfico 5. Quanto à presença de dificuldade durante a amamentação, as puérperas apresentaram uma intensidade de dor não muito forte, ou seja, apenas $20 \%$ relataram senti-la. Com relação à dificuldade de ir ao banheiro, um percentual de $25 \%$ referiu dor durante à micção. Ao permanecer na posição de repouso (decúbito dorsal), somente $35 \%$ relataram ter sentido algum grau de limitação.

\section{Discussão}

Conforme Varella (2011), no pós-operatório de cesariana, a dor é caracterizada como aguda, ou seja, apresenta início súbito com término previsível, e está intimamente ligada a dano tecidual devido às reações inflamatórias decorrentes de um processo traumático, que produzem a dor.

Melo et al. (2006) relatam, que é no segundo dia após a cirurgia de cesariana, que ocorre a metabolização das drogas anestésicas usadas na anestesia e é um período também onde há um aumento da movimentação da mãe com o intuito de cuidar do recém-nascido e dela própria, provavelmente aumentando assim a dor.

Os resultados do presente estudo corroboram com os de Navarro e Pacheco (2000) e Nuñez e Carrasco (2000), que verificaram a eficácia da EENT em associação ou não com outros recursos farmacológicos que proporcionam analgesia.

Sousa et al. (2009), ao analisar o nível de desconforto em 60 puérperas, observaram um escore de dor de $45 \%$ para atividade de ir ao banheiro confrontando assim o presente estudo, pois, de acordo com este, apenas $25 \%$ referiram sentir dor durante a micção. 
O presente estudo assemelha-se com o de Tonella, Araújo e Silva (2006) que ao realizar um estudo com uso da EENT para redução da dor pós-operatória, utilizou os eletrodos dispostos de forma peri-incisional cruzada, onde a duração do tratamento foi de 30 minutos, e após a intervenção, avaliouse novamente o escore da dor por meio da EVA.

Segundo Bjordal et al. (2003) em alguns casos, o uso da corrente da EENT juntamente com o da fita adesiva e do gel podem causar irritações na pele. Diferente dos achados deste estudo, não foi encontrada qualquer tipo de alterações cutâneas relacionadas ao seu uso.

\section{Conclusão}

De acordo com os resultados deste estudo, a EENT mostrou-se ser eficaz, pois reduziu significativamente os escores da dor causados pela lesão tecidual resultantes do procedimento cirúrgico no período de puerpério imediato.

Dentre as funções limitadas mais relatadas, encontra-se a de sentar e levantar da cama e realizar a mudança de decúbito dorsal para decúbito lateral representando $90 \%$ das mulheres, assim também como o ato de tossir, que caracterizou $85 \%$ da dor.

Considerando que o questionário para caracterizar as principais limitações de atividades foi utilizado somente antes da intervenção, sugiro que ele seja aplicado em futuras pesquisas após a utilização da EENT, com o intuito de avaliar se as limitações permaneceram ou desaparecem após o seu uso.

\section{Referências}

BAVARESCO, G. Z. et al. O Fisioterapeuta como Profissional de Suporte à Parturiente. Ciência e Saúde Coletiva.vol.16, n.7. 2011.

BJORDAL, J.M. et al. Transcutaneous electrical nerve stimulation (TENS) can reduce postoperative analgesic consumption. A meta analysis with assessment of optimal treatment parameters for postoperative pain. Ann Eur J Pain 2003; 7: 181-8.

FERRAZ, F.S; MOREIRA, C. M. C. Eletroanalgesia com utilização da TENS no pós- operatório de cirurgia cardíaca. Fisioterapia em Movimento. Curitiba. vol. 22, n. 1, 2009. 
FERREIRA, C.H.J; BELEZA, A.C.S. Abordagem fisioterapêutica na dor pós- operatória: a Eletroestimulação Nervosa Transcutânea (ENT). Rev. Col. Bras. Cir. Ribeirão Preto. vol. 34, n. 2, 2007.

MACIEL, C.C; CAMARA, S. M. A, 2008. Influência da Estimulação Elétrica Nervosa Transcutânea (TENS) associada ao alongamento muscular no ganho de flexibilidade. Revista Brasileira de Fisioterapia. vol.12, n. 5, 2008.

MELO, P. G. et al. Estimulação Elétrica Nervosa Transcutânea (TENS) no pós- operatório de cesariana. Revista Brasileira de Fisioterapia. vol.10, n. 2, 2006.

NAVARRO, N.C; PACHECO, C.M. Transcutaneous electric stimulation (TENS) to reduce pain after cesarean section. Ann Ginecol Obstet Mex 2000; 68: 60-3.

NUÑEZ, C.N; CARRASCO, M. F. P. Estimulatión eléctrica transcutánea (EET) para reducir El dolor después de lá cesárea, Ginecologya y obstetrícia de Mexico, v. 68, p.60-63, 2000.

SANTANA, L. S et al. Utilização dos recursos fisioterapêuticos no puerpério: revisão da literatura. FEMINA. vol. 39, n.5, 2011.

SOUSA, L. et al. Mensuração e características de dor após cesárea e sua relação com limitação de atividades. Acta Paul Enfer. vol. 22, n. 6, 2009.

TONELlA, R. M; ARAÚJO, S; SILVA, A. M. O. Estimulação Elétrica Nervosa Transcutânea no Alívio da Dor Pós-Operatória Relacionada com Procedimentos Fisioterapêuticos em Pacientes Submetidos a Intervenções Cirúrgicas Abdominais. Rev. Bras. Anestesiol. vol.56, n.6, Campinas, 2006.

TELLES, G. F et al. A TENS e sua vertentes de aplicação tradicionais e contemporâneas. Um estudo de revisão. Revista Digital. vol. 15, n.152, Buenos Aires, 2011.

RETT, M. T. et al. Atendimento de puérperas pela fisioterapia em uma maternidade pública humanizada. Fisioterapia e Pesquisa. vol. 15, n. 4, 2008. 
http://idonline.emnuvens.com.br/id

ISSN on-line: 1981-1179

VARELLA, R.S.Q. Avaliação na dor do pós-operatório de cesariana através da utilização do questionário de MCGILL. 2011. 92 f. Dissertação de mestrado- FIOCRUZ. Rio de Janeiro.

\section{Como citar este artigo (Formato ISO):}

SANTANA, F.M.; CAVALCANTE, E.G.; MATIAS, D.M.G.; OLIVEIRA, A.A.G. Benefícios da Estimulaçao Elétrica Nervosa Transcutânea para a redução da dor no pós-operatório da cesariana e sua relação com as limitações de atividade. Id on Line Revista de Psicologia, Fevereiro de 2014, vol.8, n.22, p. 95-104. ISSN 1981-1189.

Recebido: 20/12/2013

Aceito: 05/02/2014 